\title{
Evaluación ecocardiográfica de la gravedad de la estenosis aórtica e inconsistencias entre los criterios diagnósticos
}

\author{
Natalia Tamayo Artunduaga ${ }^{a, *}$, Alexander Londoño Cadavid ${ }^{a}$, \\ Karen Estupiñán ${ }^{\mathrm{b}}$, Jaime Luis López Torres ${ }^{\mathrm{b}}$ y Gustavo Restrepo Molina ${ }^{\mathrm{b}}$
}

\author{
a Departamento de Ecocardiografía, Universidad CES, Medellín, Colombia \\ b Departamento de Ecocardiografía, Clínica Medellín, Medellín, Colombia
}

Recibido el 26 de junio de 2015; aceptado el 17 de diciembre de 2015

Disponible en Internet el 18 de febrero de 2016

\section{PALABRAS CLAVE \\ Estenosis aórtica; \\ Ecocardiografía; \\ Diagnóstico}

\begin{abstract}
Resumen
Objetivo: Se ha reportado discrepancia en el grado de severidad de la estenosis aórtica por la ecocardiografía basado en las guías. Exploramos las inconsistencias entre los parámetros doppler y el área valvular aórtica en grupos de pacientes con anillo aórtico: pequeño, promedio y grande.

Métodos: Análisis transversal retrospectivo de ecocardiografías desde los años 2010 hasta el 2014 de los pacientes con: estenosis aórtica, fracción de eyección $\geq 50 \%$, gradiente medio $\geq$ $20 \mathrm{mmHg}$ y área valvular aórtica $\leq 1,5 \mathrm{~cm}^{2}$, divididos en tres grupos: anillo aórtico pequeño $1,3-1,8 \mathrm{~cm}$, anillo aórtico promedio $1,9-2,2 \mathrm{~cm}$ y anillo aórtico grande mayor de $2,3 \mathrm{~cm}$.

Resultados: De 245 pacientes, el 47\% hombres, presentaban el área valvular aórtica por continuidad (AVA) $0,84 \pm 0,29 \mathrm{~cm}^{2}$ y el gradiente medio fue de $34,8 \mathrm{mmHg}$. La estenosis aórtica paradójica con bajo flujo bajo el gradiente se presentó en el 11,8\%. La correlación más fuerte fue entre el anillo aórtico y la talla. El punto de corte del AVA de $1 \mathrm{~cm}^{2}$ fue consistente para pacientes con el anillo aórtico promedio y grande. El índice doppler fue el parámetro con la correlación más fuerte con el AVA en todos los grupos. La indexación de AVA por el área de superficie corporal y la talla no mejoró la discrepancia en pacientes con el anillo aórtico pequeño.

Conclusiones: Los parámetros que evalúan el grado de gravedad de la estenosis aórtica basados en las guías actuales son consistentes en pacientes con el anillo aórtico grande y promedio. El punto de corte para la estenosis aórtica es variable y dependiente del anillo aórtico y debe considerar revisión de puntos de corte en las guías para pacientes con el anillo aórtico pequeño. (c) 2016 Sociedad Colombiana de Cardiología y Cirugía Cardiovascular. Publicado por Elsevier España, S.L.U. Este es un artículo Open Access bajo la CC BY-NC-ND licencia (http:// creativecommons.org/licencias/by-nc-nd/4.0/).
\end{abstract}

\footnotetext{
* Autor para correspondencia.

Correo electrónico: natamayo@gmail.com (N. Tamayo Artunduaga).
} 


\section{KEYWORDS}

Aortic stenosis; Echocardiogram; Diagnosis

\section{Echocardiographic evaluation of aortic stenosis severity and inconsistencies} amongst diagnostic criteria

\begin{abstract}
Motivation: Discrepancies have been reported in the degree of severity of aortic stenosis assessed by means of an echocardiogram based on the guidelines. We explore inconsistencies of doppler parametres and aortic valve area in patient groups with a small, average and large aortic ring.

Methods: Retrospective, cross-sectional analysis of echocardiograms between 2010 and 2014 in patients with aortic stenosis, ejection fraction $\geq 50 \%$, average gradient $\geq 20 \mathrm{mmHg}$ and aortic valve area $\leq 1.5 \mathrm{~cm}^{2}$, were divided into three groups: small aortic ring $1.3-1.8 \mathrm{~cm}$, average aortic ring 1.9-2.2 cm and large aortic ring, larger than $2.3 \mathrm{~cm}$.

Results: Out of 245 patients, $47 \%$ of whom were men, presented the aortic valve area (AVA) continuity $0.84 \pm 0.29 \mathrm{~cm}^{2}$ and the average gradient was $34.8 \mathrm{mmHg}$. Paradoxical low-flow, low-gradient aortic stenosis was present in $11.8 \%$ of the cases. Strongest correlation was found between the aortic ring and the size. AVA cut point of $1 \mathrm{~cm}^{2}$ was consistent for patients with an average and large aortic ring. Doppler index was the parametres with the highest correlation with AVA in all groups. The AVA indexing according to the body surface and not the size did not improve discrepancies for patients with small aortic rings.

Conclusions: Parametres that assess the degree of severity of aortic stenosis based on current guidelines are consistent for patients with a large and average aortic ring. Aortic stenosis cut point varies and depends on the aortic ring; a review of the guidelines of the cut points for patients with a small aortic ring should be considered.

(C) 2016 Sociedad Colombiana de Cardiología y Cirugía Cardiovascular. Published by Elsevier España, S.L.U. This is an open access article under the CC BY-NC-ND license (http:// creativecommons.org/licenses/by-nc-nd/4.0/).
\end{abstract}

\section{Introducción}

La estenosis aórtica es la enfermedad valvular más frecuente referida para el manejo quirúrgico con una prevalencia en aumento debido al envejecimiento de la población, se presenta en el $0,2 \%$ de los adultos entre 50 a 59 años de edad y aumenta hasta el $9,8 \%$ en octogenarios y su prevalencia general es del 2,8\% en mayores de 75 años de edad $^{1}$. A pesar de las recomendaciones de las guías internacionales $A H A / A C C F$ y ESC ${ }^{2,3}$, para la clasificación de la severidad de la estenosis aórtica, aún quedan interrogantes por resolver acerca de los parámetros ecocardiográficos que mejor definen su gravedad, especialmente, los parámetros como el área valvular aórtica indexada, considerando la heterogeneidad antropométrica en nuestra población, con respecto a las poblaciones de las cuales se han derivado estos parámetros. Se han encontrado inconsistencias en el grado de severidad de la estenosis aórtica por la ecocardiografía en 1 de cada 3 pacientes y por el cateterismo en 1 de 4 pacientes $^{4,5}$.

Se ha considerado que una fuente importante de inconsistencias es la medición del anillo aórtico o tracto de salida del ventrículo izquierdo, el cual se incluye en la ecuación de continuidad para calcular el área valvular aórtica ${ }^{6}$. Este último parámetro es considerado de importancia para el diagnóstico de la estenosis aórtica y definir su pronóstico.

Varios autores han sugerido un punto de corte del área valvular aórtica menor de $0,8 \mathrm{~cm}^{2}$ para definir la estenosis aórtica grave en comparación el punto de corte de las guías actuales menor o igual a $1,0 \mathrm{~cm}^{2}$, que tiene una sensibilidad del $95 \%$ y especificidad del $75 \%$, sobreestimando la gravedad de la estenosis aórtica cuando se compara con la velocidad máxima o el gradiente medio transvalvular ${ }^{7}$.

En este caso se exploró el nivel de correlación entre los diferentes parámetros para evaluar la severidad de la estenosis aórtica y la influencia de la indexación por la superficie corporal y la talla en nuestra población y evaluamos la medición del anillo aórtico como fuente de inconsistencias en la evaluación de la severidad.

\section{Métodos}

Se revisó en forma retrospectiva la base de datos de los ecocardiogramas transtorácicos realizados entre enero de 2010 y febrero de 2014 en el laboratorio de ecocardiografía de la Clínica Medellín. Se incluyeron pacientes mayores de 18 años de edad, con diagnóstico de estenosis aórtica, fracción de eyección mayor del $50 \%$, gradiente medio mayor de $20 \mathrm{mmHg}$, área valvular aórtica menor de $1,5 \mathrm{~cm}^{2}$, insuficiencia aórtica menos que moderada, insuficiencia mitral menos que moderada y frecuencia cardiaca entre 60-90 latidos por minuto. Se excluyeron pacientes críticos en la unidad de cuidado intensivo, estudios de ecocardiografía transesofágica, válvulas protésicas, estudios con limitaciones de la ventana acústica que no permitían valoración adecuada de la patología valvular aórtica y los que no cumplían criterios de inclusión.

\section{Ecocardiografía}

Todos los pacientes incluidos fueron evaluados con la ecocardiografía transtorácica bidimensional. Se calculó la fracción 
de eyección del ventrículo izquierdo por el método de Simpson biplano o estimación visual. Se midió el anillo aórtico (tracto de salida del ventrículo izquierdo) en el eje largo paraesternal con zoom, de borde interno a borde interno en el punto de inserción de los velos de la válvula aórtica.

Las mediciones doppler fueron obtenidas según las recomendaciones ASE (Asociación Americana de Ecocardiografía $)^{8}$.

Además, se calculó el índice de pérdida de energía ${ }^{9}$ y la impedancia válvulo-arterial ${ }^{10}$ como parámetros adicionales.

El área valvular aórtica calculada por ecuación de continuidad fue indexada por superficie corporal y talla.

El anillo aórtico se clasificó como pequeño entre 1,3 $1,8 \mathrm{~cm}$, mediano entre $1,9-2,2 \mathrm{~cm}$ y grande entre $2,3-$ $2,8 \mathrm{~cm}$, teniendo en cuenta la distribución estándar en nuestra población. En cada grupo se evaluó la correlación entre el área valvular aórtica como parámetro dependiente de la medición del anillo aórtico con otros parámetros de severidad.

Además, se evalúo el área valvular aórtica calculada por ecuación de continuidad indexada por la talla (no se incluyó la talla elevada a la 2,7) y por el área de la superficie corporal ASC (calculada con la fórmula de Dubois) y su correlación con otros parámetros de gravedad. Se usaron como criterios de severidad los definidos en las guías de enfermedad valvular de la Asociación Americana del Corazón (AHA) del año $2014^{2}$, para la estenosis aórtica severa: velocidad máxima de la válvula aórtica $>400 \mathrm{~cm} / \mathrm{s}$, gradiente medio $<40 \mathrm{mmHg}$, área valvular aórtica $<1 \mathrm{~cm}^{2}$, área valvular aórtica indexada $\mathrm{ASC}<0,6 \mathrm{~cm}^{2} / \mathrm{m}^{2}$ y relación de integrales (índice de velocidades doppler) $<0,25$.

\section{Análisis estadístico}

Para el análisis descriptivo se aplicaron: las frecuencias absolutas, las relativas y los indicadores de resumen, como: la media, la desviación estándar, la mediana y el rango intercuartílico. Se estableció el criterio de normalidad por medio de la prueba de Shapiro Wilk y el criterio de homogeneidad de las varianzas por la prueba de Levene; y con base en estas pruebas se realizó el análisis de varianza (ANOVA), para la diferencias de los parámetros ecocardiográficos según grupo de tamaño de anillo aórtico y las diferencias de edad entre los grupos se ajustaron con un análisis de covarianza (ANCOVA), aplicando la prueba de comparación múltiple con el método de Scheffé; de igual manera se empleó la prueba de Kruskal Wallis con la prueba de comparación van der Waerden cuando fue necesario. Para establecer la relación lineal entre las variables continuas se aplicó regresión lineal simple y se reportó el coeficiente de correlación de Pearson o el coeficiente de Spearman y el coeficiente de determinación. Las variables categóricas se compararon entre los tres grupos de tamaño del anillo aórtico utilizando la prueba $\chi^{2}$ de independencia de Pearson. Para ajustar y explicar la posible relación entre el anillo aórtico y algunas variables demográficas, antropométricas y de la ecocardiografía, se aplicó un modelo de regresión lineal múltiple. Un valor de $\mathrm{p}<0,5$ se consideró estadísticamente significativo. Los datos fueron analizados en los software Aabel 20/20 data vision 3 y en el Stata 13.

\section{Resultados}

En el período evaluado se realizaron 17.640 estudios ecocardiográficos, de los cuales 245 cumplieron con criterios de inclusión, el $53 \%$ fueron mujeres y el $47 \%$ hombres, la mayoría fueron pacientes mayores de 60 años de edad $(\mathrm{N}=194$, $79 \%$ ), el $15,5 \%$ presentaron sobrepeso, el $5 \%$ con obesidad grado 1 y el $0,8 \%$ con obesidad grado 2 . Las mujeres tenían con mayor frecuencia: obesidad, menor talla y con mayor frecuencia los anillos aórticos pequeños con diferencias significativas con respecto a los hombres $(p<0,00001)$. La mediana de la fracción de eyección fue de $65 \% \pm 5$, sin diferencias significativas entre los diferentes grupos $(p=0,47)$.

Nuestro estudio mostró que el $70,6 \%$ de los pacientes tenían: el diámetro del anillo aórtico mediano, anillo grande el $16,3 \%$ y la minoría anillo pequeño (13\%). Las mujeres tenían con mayor frecuencia anillos pequeños (16\%) vs. hombres $(9,5 \%) p<0,0001$.

El anillo aórtico promedio fue 2,01 $\pm 0,25$, el área valvular aórtica por continuidad (AVA) 0,84 $\pm 0,29 \mathrm{~cm}^{2}$, el área valvular indexada por el área de superficie corporal (AVAi) $0,51 \pm 0,18 \mathrm{~cm}^{2} / \mathrm{m}^{2}$. La mediana del gradiente medio fue $34,8 \mathrm{mmHg}$, velocidad máxima $378 \mathrm{~cm} / \mathrm{s}$ y la relación de integrales 0,26 , tabla 1.

Se hallaron 145 pacientes (59\%) con el área valvular aórtica menor de $1 \mathrm{~cm}^{2}$, de los cuales 67 (46\%) tenían el gradiente medio menor de $40 \mathrm{mmHg}$ y 29 (43\%), tenían bajo flujo (volumen sistólico $<35 \mathrm{ml} / \mathrm{m}^{2}$ ), configurando el diagnóstico de estenosis aórtica bajo flujo, bajo gradiente con fracción de eyección preservada en el 11,8\% de la población.

La impedancia válvulo-arterial (Zva) evidenció ser mayor de $4,5 \mathrm{mmHg} / \mathrm{ml} . \mathrm{m}^{2}$ en el $45 \%$ de los pacientes $(p<0,00001)$ como hallazgo esperado.

El índice de pérdida de energía indexado por el área de la superficie corporal menor de $0,6 \mathrm{~cm}^{2} / \mathrm{m}^{2}$ fue más frecuente en los pacientes con anillo aórtico pequeño en el $84,4 \%$, en comparación con medianos $(43,9 \%)$ y grandes $(27,5 \%)$. tabla 2.

\section{Correlación entre las medidas antropométricas y el tamaño del anillo aórtico por 2D}

No se halló buena correlación entre ASC y el diámetro del anillo aórtico $\left(R_{S}=0,27, p=0,00001\right.$; fig. $\left.1 A\right)$. La correlación fue ligeramente mayor entre la talla y el diámetro del anillo aórtico (Rs =0,37, $p=0,0000$; fig. 1B). No se encontraron diferencias significativas según el género.

Correlación entre AVA, AVA/ASC y AVA/talla con otros parámetros de gravedad sugeridos por las guías

El AVA en relación con la velocidad máxima de la válvula aórtica demuestra variación significativa entre los diferentes grupos según el tamaño del anillo, hallando mayor correlación en el grupo de pacientes con anillo grande $\left(R_{S}=-0,66, p=0,0000\right)$ y mediano $\left(R_{S}=-0,60, p=0,0000\right)$, fig. 2.

El AVA indexada por ASC y talla mostró moderada correlación con la velocidad máxima de la válvula aórtica en pacientes con el anillo mediano y grande $\left(R_{S}=-0,5, p=\right.$ $0,0000)$ y débil en pacientes con el anillo pequeño $\left(R_{S}=-0,4\right.$, $p=0,0000)$. 
Tabla 1 Características demográficas y ecocardiográficas según tamaño del anillo aórtico

\begin{tabular}{|c|c|c|c|c|c|}
\hline Variable & $\begin{array}{l}\text { Anillo Aórtico } \\
(\mathrm{n}=245)\end{array}$ & $\begin{array}{l}\text { Anillo Aórtico } \\
\text { Pequeño }(n=32)\end{array}$ & $\begin{array}{l}\text { Anillo Aórtico } \\
\text { Medio }(n=173)\end{array}$ & $\begin{array}{l}\text { Anillo Aórtico } \\
\text { Grande }(n=40)\end{array}$ & Valor $\mathrm{p}$ \\
\hline Edad (años) & 73 (19) & $78(20)$ & 73 (17) & $64(24)$ & 0,0005 \\
\hline Peso (k) & $64(17,5)$ & $58(17)$ & $63(17)$ & $68(12)$ & 0,0193 \\
\hline Talla $(\mathrm{cm})$ & $158,6 \pm 8,65$ & $155.6 \pm 8,68$ & $157,9 \pm 8,31$ & $164,2 \pm 7,56$ & $<0,00001$ \\
\hline $\operatorname{IMC}\left(k / m^{2}\right)$ & $25(6)$ & $24(5)$ & $25(6)$ & $26(5)$ & 0,6523 \\
\hline Área superficie corporal $\left(\mathrm{m}^{2}\right)$ & $1,66 \pm 0,186$ & $1,61 \pm 0,185$ & $1,65 \pm 0,179$ & $1,76 \pm 0,184$ & $0,002^{* *}$ \\
\hline Presión sistólica (mmHg) & $120(30)$ & $124(20)$ & $120(30)$ & $120(39)$ & 0,4343 \\
\hline Presión diastólica (mmHg) & $70(20$ & $70(24)$ & $70(20)$ & $70(20)$ & 0,1694 \\
\hline Frecuencia cardiaca (lat/min) & $75,5 \pm 13,3$ & $76 \pm 11,5$ & $76,4 \pm 14$ & $72,3 \pm 10,4$ & $0,305^{*}$ \\
\hline Anillo & $4(1,2)$ & $2,75(0,6)$ & $4(0,8)$ & $5,7(1)$ & $<0,0001$ \\
\hline Volumen Sistólico $(\mathrm{ml})$ & $72,2(31,25)$ & $47,7(25)$ & $71,8(27)$ & $95(24,2)$ & $<0,0001$ \\
\hline $\begin{array}{l}\text { Volumen Sistólico indexado } \\
\qquad\left(\mathrm{ml} / \mathrm{m}^{2} \mathrm{SC}\right)\end{array}$ & $45,5 \pm 13,6$ & $31,8 \pm 11,6$ & $45,6 \pm 11,6$ & $56 \pm 12,3$ & $<0,0000^{* *}$ \\
\hline Área valvular aórtica AVA $\left(\mathrm{cm}^{2}\right)$ & $0,8(0,4)$ & $0,5(0,2)$ & $0,85(0,3)$ & $1,1(0,4)$ & $<0,0001^{* \star *}$ \\
\hline Estenosis grave $(n=157)$ & $0,7(0,3)$ & $0,5(0,2)$ & $0,7(0,2)$ & $0,8(0,1)$ & $<0,001$ \\
\hline Estenosis moderada $(n=87)$ & $1,1(0,3)$ & N.A & $1,1(0,3)$ & $1,2(0,2)$ & 0,048 \\
\hline $\begin{array}{l}\text { Área valvular aórtica indexada } \\
\qquad\left(\mathrm{cm}^{2} / \mathrm{m}^{2} \mathrm{SC}\right)\end{array}$ & $0,51 \pm 0,18$ & $0,34 \pm 0,19$ & $0,53 \pm 0,21$ & $0,61 \pm 0,27$ & $<0,00001^{* *}$ \\
\hline Estenosis Grave $(n=143)$ & $0,39 \pm 0,09$ & $0,33 \pm 0,11$ & $0,39 \pm 0,09$ & $0,45 \pm 0,07$ & $<0,0001$ \\
\hline Estenosis Moderada $(\mathrm{n}=96)$ & $0,67 \pm 0,08$ & $0,67 \pm 0,11$ & $0,66 \pm 0,08$ & $0,70 \pm 0,07$ & 0,104 \\
\hline AVA/talla & $0,532 \pm 0,18$ & $0,354 \pm 0,12$ & $0,545 \pm 0,17$ & $0,649 \pm 0,16$ & $<0,00001^{* *}$ \\
\hline AVA/IMC & $0,034(0,0175)$ & $0,021(0,0098)$ & $0,034(0,0160)$ & $0,045(0,0145)$ & $<0,0001^{* * *}$ \\
\hline $\begin{array}{l}\text { Relación integrales aórtica } \\
\text { (VTIpw/VTIcw) }\end{array}$ & $0,262 \pm 0,077$ & $0,261 \pm 0,095$ & $0,269 \pm 0,076$ & $0,237 \pm 0,060$ & $0,2232^{*}$ \\
\hline Estenosis Grave $(n=106)$ & $0,193 \pm 0,034$ & $0,191 \pm 0,039$ & $0,194 \pm 0,033$ & $0,191 \pm 0,034$ & 0,947 \\
\hline Estenosis moderada $(n=138)$ & $0,313 \pm 0,053$ & $0,323 \pm 0,064$ & $0,315 \pm 0,054$ & $0,293 \pm 0,029$ & 0,168 \\
\hline $\begin{array}{l}\text { Impedancia valvuloarterial } \\
\qquad\left(\mathrm{mmHg} / \mathrm{ml} / \mathrm{m}^{2}\right)\end{array}$ & $3,7(1,75)$ & $5,3(3,1)$ & $3,6(1,5)$ & $2,9(1,1)$ & $<0,0001^{* * *}$ \\
\hline Estenosis Grave $(n=112)$ & $2,9(0,8)$ & N.A & $2,9(0,9)$ & $2,7(0,8)$ & 0,090 \\
\hline Estenosis moderada $(n=60)$ & $3,9(0,5)$ & $3,9(0,4)$ & $3,9(0,5)$ & $4,1(0,5)$ & 0,842 \\
\hline $\begin{array}{l}\text { Índice pérdida indexado } \\
\qquad\left(\mathrm{cm}^{2} / \mathrm{m}^{2}\right)\end{array}$ & $0,7(0,4)$ & $0,4(0,2)$ & $0,7(0,4)$ & $1(0,5)$ & $<0,0001^{n * * *}$ \\
\hline Estenosis Grave $(n=114)$ & $0,4(0,2)$ & $0,4(0,3)$ & $0,4(0,2)$ & $0,5(0,1)$ & 0,016 \\
\hline Estenosis moderada $(n=131)$ & $0,9(0,3)$ & $0,7(0,4)$ & $0,9(0,4)$ & $1(0,3)$ & 0,015 \\
\hline Aorta ascendente & $3,3(0,7)$ & $3,1(0,5)$ & $3,2(0,5)$ & $3,6(0,6)$ & 0,0005 \\
\hline Raíz aórtica $(\mathrm{cm})$ & $3,149 \pm 0,467$ & $3,027 \pm 0,436$ & $3,067 \pm 0,442$ & $3,523 \pm 0,418$ & $<0,00001^{* *}$ \\
\hline $\begin{array}{l}\text { Velocidad tracto de salida } \mathrm{VI} \\
\qquad(\mathrm{cm} / \mathrm{seg})\end{array}$ & $96(24,5)$ & $98,5(23)$ & $98(27)$ & $86(15,5)$ & 0,0010 \\
\hline $\begin{array}{l}\text { Velocidad máxima válvula } \\
\text { aórtica (cm/seg) }\end{array}$ & $378(97)$ & $385(137)$ & $371(97)$ & $373(125)$ & 0,8422 \\
\hline Estenosis Grave $(\mathrm{n}=96)$ & $451,5(82,5)$ & $506,5(98)$ & $447,5(77)$ & $472(58)$ & 0,144 \\
\hline Estenosis moderada $(n=149)$ & $347(52)$ & $351(35)$ & $348(56)$ & $342(54)$ & 0,726 \\
\hline Gradiente medio aórtico $(\mathrm{mmHg})$ & 34. $8(19,5)$ & $35,5(25,3)$ & $33(19)$ & $36(31)$ & 0,9471 \\
\hline Estenosis Grave $(n=85)$ & $53(22)$ & $70(16,5)$ & $51(19)$ & $60(17)$ & 0,064 \\
\hline Estenosis moderada $(n=157)$ & $29(10)$ & $30(10)$ & $30(8)$ & $26(13,5)$ & 0,348 \\
\hline Fracción de Eyección VI (\%) & $65(5)$ & $65(1,3)$ & $65(5)$ & $65(5)$ & 0,4759 \\
\hline
\end{tabular}

Los valores se presentan en: media aritmética \pm desviación estándar o mediana (Rango intercuartílico). N.A: No Aplica insuficiente ( $\mathrm{n}$ ). " ANCOVA ajustado por edad.

** ANCOVA ajustado por edad con comparación múltiple, método de Scheffé: $p<0,0001$ entre el anillo aórtico pequeño y el anillo aórtico grande; y entre el anillo aórtico mediano y el anillo aórtico grande.

Kruskal-Wallis con comparación de la prueba van der Waerden: $p<0,0001$ entre el anillo aórtico pequeño y el anillo aórtico grande; entre el anillo aórtico mediano y el anillo aórtico grande; y entre el anillo aórtico pequeño y el anillo aórtico mediano.

${ }^{* * * *}$ Kruskal-Wallis con comparación de la prueba van der Waerden: $p<0,0001$ entre el anillo aórtico pequeño y el anillo aórtico grande; y entre el anillo aórtico pequeño y el anillo aórtico mediano. $\mathrm{P}<0,001$ entre el anillo aórtico mediano y el anillo aórtico grande. 
Tabla 2 Aspectos demográficos, antropométricos y ecocardiográficos según el tamaño del anillo aórtico

\begin{tabular}{|c|c|c|c|c|c|c|}
\hline Variable & Categoría & $\begin{array}{l}\text { Anillo } \\
\text { Aórtico } \\
\text { Total n (\%) }\end{array}$ & $\begin{array}{l}\text { Anillo } \\
\text { Aórtico } \\
\text { Pequeño n } \\
\text { (\%) }\end{array}$ & $\begin{array}{l}\text { Anillo } \\
\text { Aórtico } \\
\text { Medio n (\%) }\end{array}$ & $\begin{array}{l}\text { Anillo } \\
\text { Aórtico } \\
\text { Grande n (\%) }\end{array}$ & Valor $\mathrm{p}$ \\
\hline \multirow[t]{2}{*}{ Género } & Hombre & $115(46,9)$ & $11(34,4)$ & $73(42,2)$ & $31(77,5)$ & $<0,00001$ \\
\hline & Mujer & $130(53,1)$ & $21(65,6)$ & $100(57,8)$ & $9(22,5)$ & \\
\hline \multirow[t]{3}{*}{ Talla $(\mathrm{cm})$} & Bajo & $131(53,5)$ & $22(68,8)$ & $98(56,6)$ & $11(27,5)$ & 0,004 \\
\hline & Medio & $112(45,7)$ & $10(31,3)$ & $74(42,8)$ & $28(70,0)$ & \\
\hline & Alto & $2(0,8)$ & $0(0,0)$ & $1(0,6)$ & $1(2,5)$ & \\
\hline \multirow{5}{*}{$\begin{array}{l}\text { Índice de masa corporal } \\
\left(\mathrm{k} / \mathrm{m}^{2}\right)\end{array}$} & Bajo peso & $2(0,8)$ & $0(0,0)$ & $2(1,2)$ & $0(0,0)$ & 0,446 \\
\hline & Normal & $114(46,5)$ & $18(56,3)$ & $79(45,7)$ & $17(42,5)$ & \\
\hline & Sobre peso & $93(38,0)$ & $11(34,4)$ & $62(35,8)$ & $20(50,0)$ & \\
\hline & Obesidad grado I & $31(12,7)$ & $2(6,3)$ & $27(15,6)$ & $2(5,0)$ & \\
\hline & Obesidad grado II & $5(2,0)$ & $1(3,1)$ & $3(1,7)$ & $1(2,5)$ & \\
\hline \multirow{3}{*}{$\begin{array}{l}\text { Área superficie corporal } \\
\left(m^{2}\right)\end{array}$} & Pequeño & $67(27,3)$ & $15(46,9)$ & $46(26,6)$ & $6(15,0)$ & 0,015 \\
\hline & Mediano & $143(58,4)$ & $15(46,9)$ & $104(60,1)$ & $24(60,0)$ & \\
\hline & Grande & $35(14,3)$ & $2(6,3)$ & $23(13,3)$ & $10(25,0)$ & \\
\hline \multirow{2}{*}{ Área valvular aórtica $\left(\mathrm{cm}^{2}\right)$} & Estenosis grave & $157(64,3)$ & $30(96,8)$ & $112(64,7)$ & $15(37,5)$ & $<0,00001$ \\
\hline & Moderada & $87(35,7)$ & $1(3,2)$ & $61(35,3)$ & $25(62,5)$ & \\
\hline Área valvular aórtica & Grave & $143(58,4)$ & $30(93,8)$ & $98(56,6)$ & $15(37,5)$ & $<0,00001$ \\
\hline \multirow{2}{*}{ indexada $\left(\mathrm{cm}^{2} / \mathrm{m}^{2}\right)$} & Moderado & $96(39,2)$ & $2(6,3)$ & $71(41,0)$ & $23(57,5)$ & \\
\hline & Leve & $6(2,4)$ & $0(0,0)$ & $4(2,3)$ & $2(5,0)$ & \\
\hline Relación integrales aórtica & Grave & $106(43,3)$ & $15(46,9)$ & $70(40,5)$ & $21(52,5)$ & 0,062 \\
\hline \multirow[t]{2}{*}{ (VTIpw/VTIcw) } & Moderado & $138(56,3)$ & $16(50,0)$ & $103(59,5)$ & $19(47,5)$ & \\
\hline & Leve & $1(0,4)$ & $1(3,1)$ & $0(0,0)$ & $0(0,0)$ & \\
\hline \multirow[t]{3}{*}{ Zva $\left(m m H g / m l / m^{2}\right)$} & Grave & $112(45,7)$ & $2(6,3)$ & $78(45,1)$ & $32(80,0)$ & $<0,00001$ \\
\hline & Moderado & $60(24,5)$ & $6(18,8)$ & $49(28,3)$ & $5(12,5)$ & \\
\hline & Leve & $73(29,8)$ & $24(75,0)$ & $46(26,6)$ & $3(7,5)$ & \\
\hline Índice de pérdida energía & Grave & $114(46,5)$ & $27(84,4)$ & $76(43,9)$ & $11(27,5)$ & $<0,00001$ \\
\hline indexado $\left(\mathrm{cm}^{2} / \mathrm{m}^{2} / \mathrm{m}^{2} \mathrm{SC}\right)$ & moderado & $131(53,5)$ & $5(15,6)$ & $97(56,1)$ & $29(72,5)$ & \\
\hline Velocidad Máxima aórtica & Grave & $96(39,2)$ & $12(37,5)$ & $68(39,3)$ & $16(40,0)$ & 0,975 \\
\hline$(\mathrm{cm} / \mathrm{seg})$ & Moderado & $149(60,8)$ & $20(62,5)$ & $105(60,7)$ & $24(60,0)$ & \\
\hline Gradiente medio aórtico & Grave & $85(34,7)$ & $9(28,1)$ & $61(35,3)$ & $15(37,5)$ & 0,717 \\
\hline \multirow{2}{*}{$(\mathrm{mmHg})$} & Moderado & $157(64,1)$ & $23(71,9)$ & $109(63,0)$ & $25(62,5)$ & \\
\hline & Leve & $3(1,2)$ & $0(0,0)$ & $3(1,7)$ & $0(0,0)$ & \\
\hline
\end{tabular}
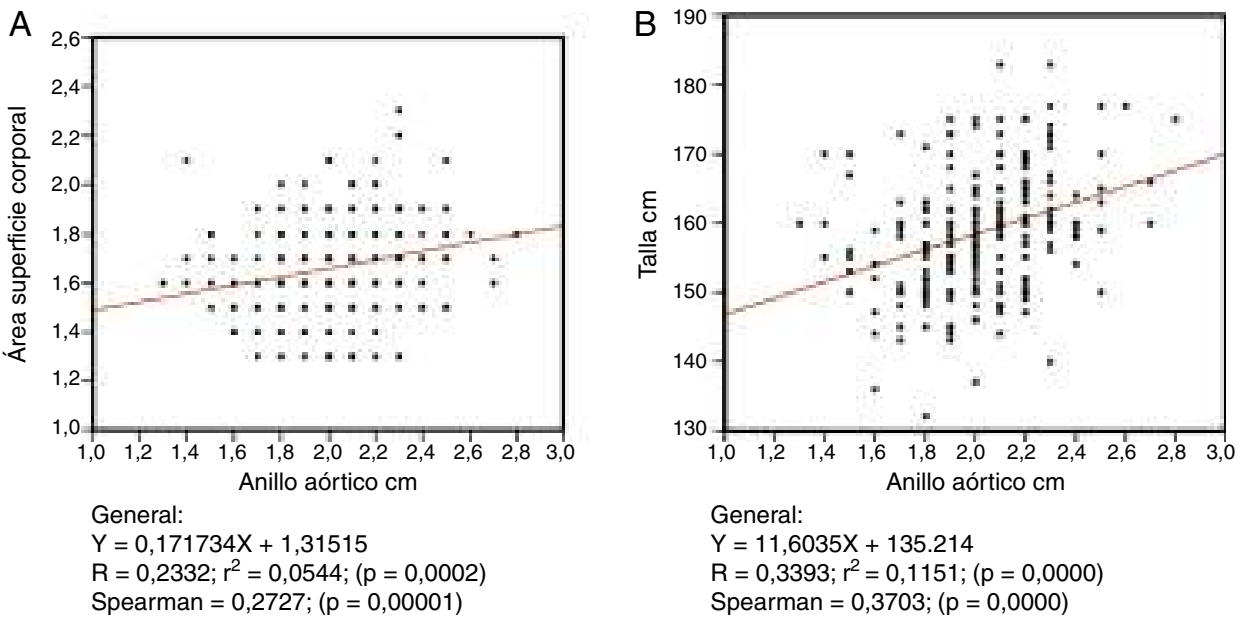

Figura 1 A Correlación entre el área de superficie corporal, la talla y el anillo aórtico.

Se halló correlación débil entre el ASC y el anillo aórtico. B La correlación fue mayor entre la talla y el anillo aórtico. 

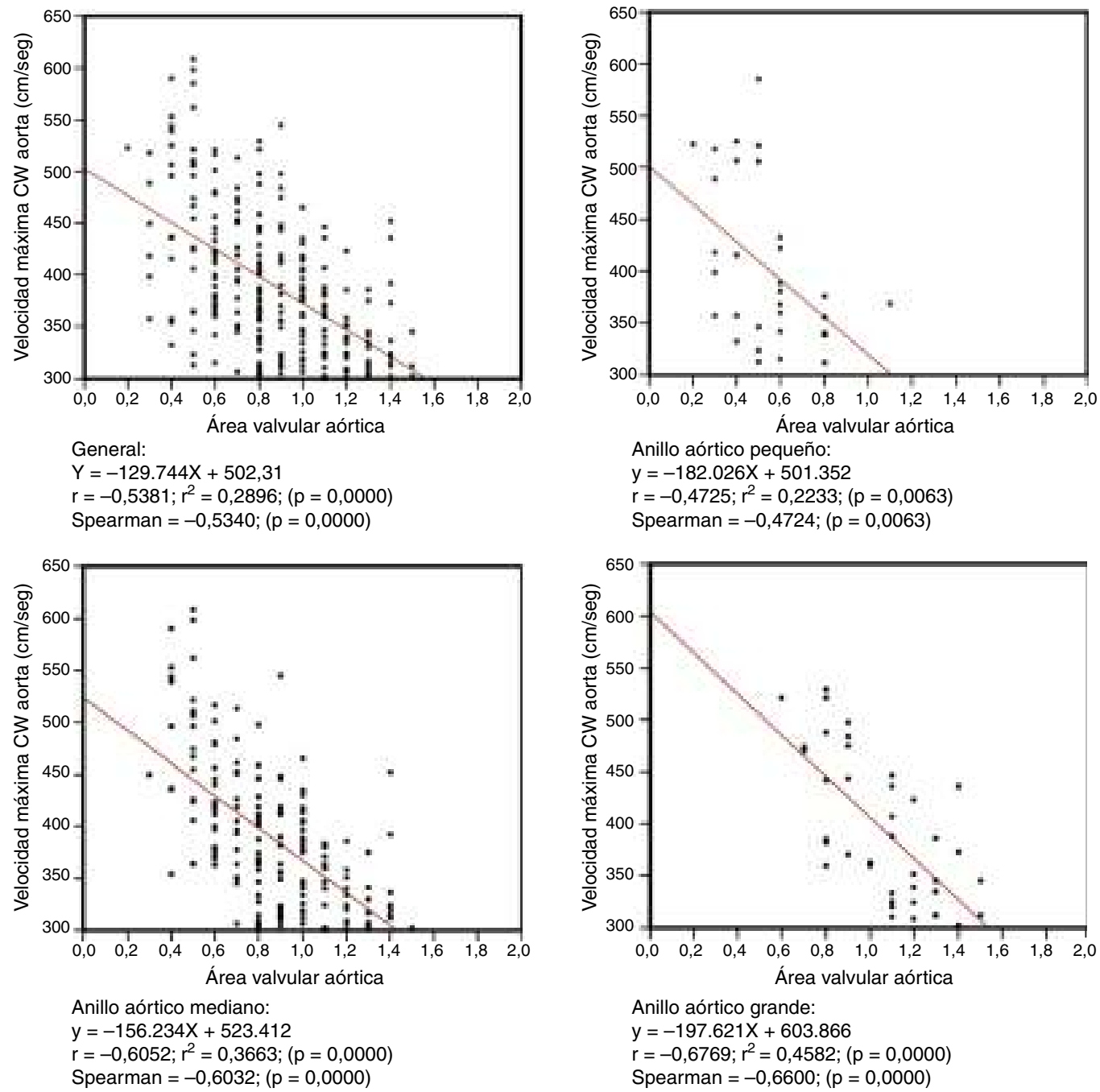

Figura 2 Correlación entre la velocidad máxima de la válvula aórtica y el área valvular aórtica.

El AVA en relación con la velocidad máxima de la válvula aórtica demuestra variación significativa entre los diferentes grupos según el tamaño del anillo, hallando correlación moderada en el grupo de pacientes con anillo grande.

La relación de integrales (índice de velocidades doppler) fue el parámetro de severidad con mayor correlación con el AVA (fig. 3) y el AVA indexada por ASC y talla (fig. 4), independiente del tamaño del anillo (todos, $R_{S}>0,7, p=0,0000$ ).

El AVA en relación con el gradiente medio demuestra variación significativa entre los diferentes grupos según el tamaño del anillo, siendo moderada la correlación en el grupo de pacientes con el anillo aórtico mediano y grande $\left(R_{S}=-0,6, p=0,0000\right)$ y débil en pacientes con el anillo aórtico pequeño, Fig. 5. El AVA indexada demostró igual correlación moderada en el grupo de pacientes con el anillo aórtico mediano y grande con respecto a los pequeños $\left(R_{S}=-0,6, p=0,0000\right)$.

\section{Discusión}

Este estudio caracteriza por primera vez en nuestra población de pacientes con la estenosis aórtica, la variabilidad de tamaño del anillo aórtico y su correlación con diferentes parámetros doppler, antropométricos y de género.
El diámetro del anillo aórtico es fundamental para el cálculo del área valvular aórtica por ecuación de continuidad y cualquier error al ser elevado al cuadrado en la ecuación afecta, significativamente, el cálculo del área valvular aórtica y es fuente de inconsistencias para determinar la gravedad de la estenosis aórtica.

Diferentes estudios han sugerido que el tamaño del anillo aórtico o tracto de salida del ventrículo izquierdo como una de las principales fuentes de inconsistencias en los parámetros de gravedad de la estenosis aórtica. Por tal motivo han sugerido en forma arbitraria la clasificación según el diámetro en el anillo pequeño, el mediano y el grande, con el fin de evaluar su influencia en determinar la gravedad ${ }^{6}$.

El diámetro del anillo aórtico mostró mejor correlación con la talla que con el área de superficie corporal, similar a lo descrito en el artículo de Michelena y Cols. ${ }^{6}$, que reportó mayor correlación entre el diámetro del tracto de salida del ventrículo izquierdo (anillo aórtico) y la talla. Como ha sido descrito en previos estudios el diámetro del anillo aórtico demostró ser menor en el grupo de mujeres ${ }^{5}$, también, se encontró diferencias significativas por género en la 

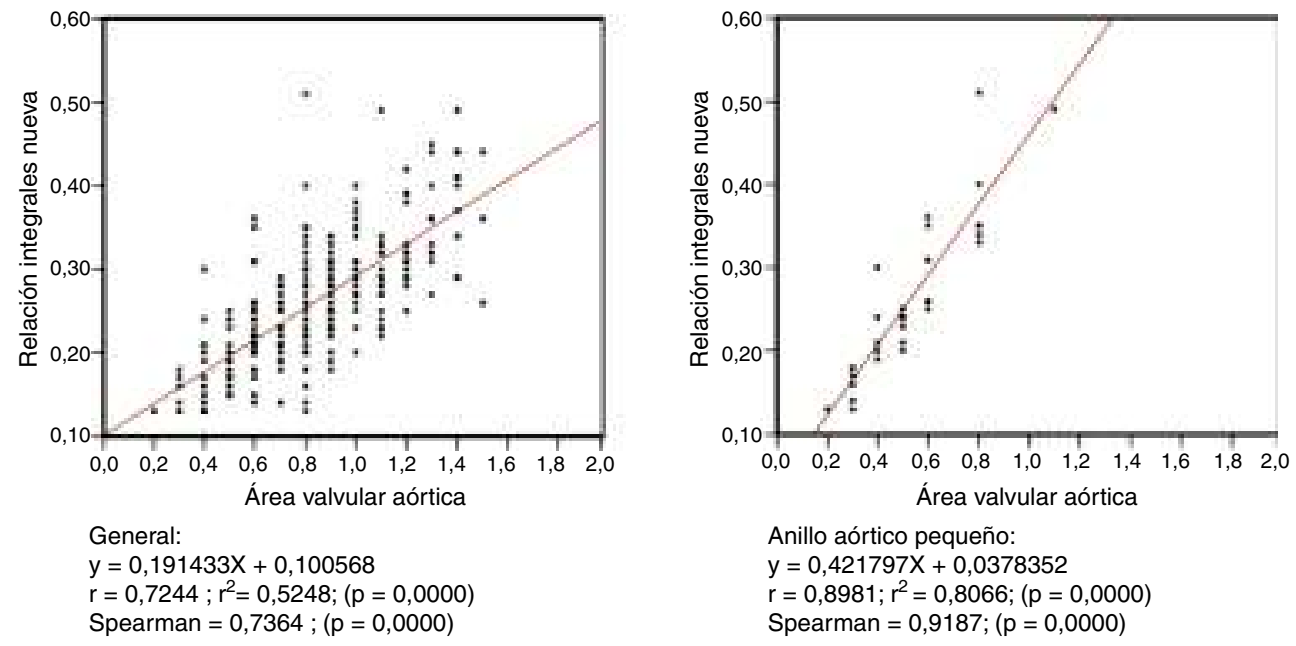

Anillo aórtico pequeño:

$y=0,421797 X+0,0378352$

$r=0,8981 ; r^{2}=0,8066 ;(p=0,0000)$

Spearman $=0,9187 ;(p=0,0000)$
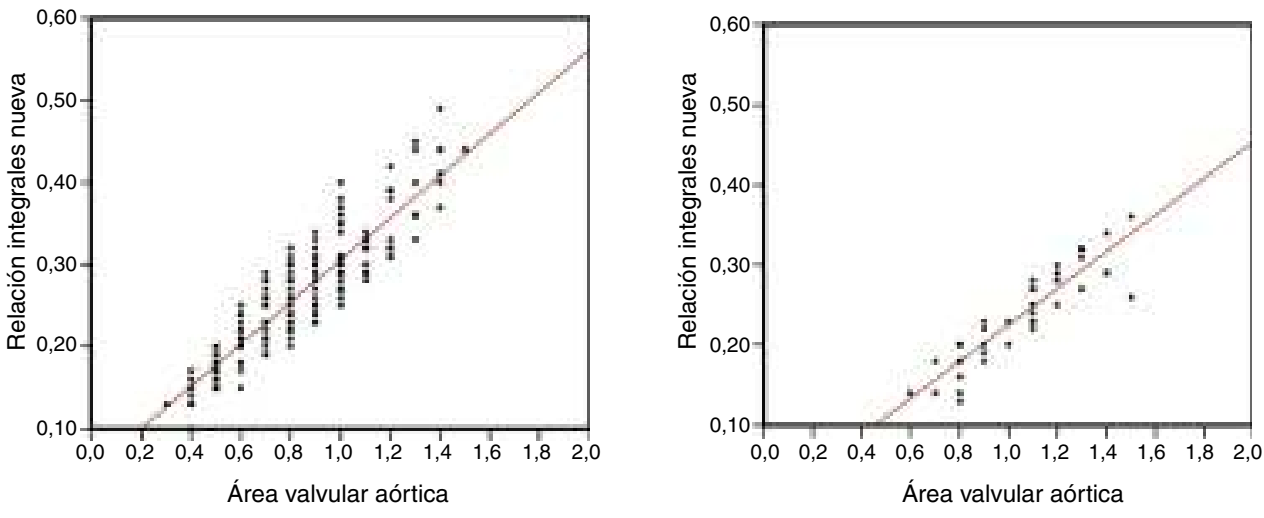

Anillo aórtico mediano:

$\mathrm{y}=0,256749 \mathrm{X}+0,0487067$

$r=0,9021 ; r^{2}=0,8138 ;(p=0,0000)$

Spearman $=0,8957 ;(p=0,0000)$

Anillo aórtico grande:

$\mathrm{y}=0,228991 \mathrm{X}-0,00437585$

$r=0,9049 ; r^{2}=0,8188 ;(p=0,0000)$

Spearman $=0,9161 ;(p=0,0000)$

Figura 3 Correlación entre la relación de integrales (índice de velocidades doppler) y el área valvular aórtica. El índice de velocidades doppler demostró fuerte correlación con el AVA, independiente del tamaño del anillo.

relación entre el diámetro del anillo aórtico y la talla, lo que resalta la importancia de considerar la indexación por talla en la estratificación de severidad de la estenosis aórtica en pacientes mujeres y/o pacientes de talla baja.

El AVA como parámetro único ha demostrado que sobreestima la severidad de la estenosis aórtica y genera inconsistencias cuando se compara con otros parámetros ${ }^{7}$, como lo descrito en nuestro estudio, en donde el 64,3 fueron clasificados en estenosis aórtica severa por AVA, en comparación con el 39,2\% y el $34.7 \%$ por velocidad máxima de la válvula aórtica y el gradiente medio transvalvular respectivamente.

En este caso se encontró que los parámetros de guías actuales son más consistentes para pacientes con el anillo aórtico grande y mediano, en estos grupos se demostró mayor correlación entre en el AVA y los diferentes parámetros de severidad (velocidad máxima de la válvula aórtica, gradiente medio e índice doppler). Otros estudios ${ }^{4,6}$, han demostrado mayor consistencia entre las guías para el grupo de pacientes con el anillo aórtico grande.

La indexación del AVA por ASC y talla no demostró ser mejor que el AVA en pacientes con el anillo pequeño. En este grupo la correlación entre AVA, AVA/ASC y AVA/talla con los otros parámetros como el gradiente medio y la velocidad máxima de la válvula aórtica fue similar, sin diferencias significativas. Probablemente, en este grupo de pacientes con el anillo pequeño el punto de corte de la guías del $\mathrm{AVA} \leq 1 \mathrm{~cm}^{2}$ es inadecuado y por esa razón no se correlacionó con los otros parámetros. Otros autores ${ }^{4,6}$, han demostrado que al disminuir el punto de corte de la estenosis aórtica $\mathrm{AVA} \leq 0,8 \mathrm{~cm}^{2}$ en estos pacientes disminuye el nivel de inconsistencias, lo que facilitaría la toma de decisiones clínicas para remitir a estos pacientes a cambio valvular aórtico o manejo médico.

Lancelotti y Cols. ${ }^{11}$, han demostrado que la velocidad máxima de la válvula aórtica mayor de $400 \mathrm{~cm} / \mathrm{s}$ está asociada con una supervivencia libre de eventos solo del $26 \%$ a 2 años. El 39\% de nuestros pacientes cumplían este criterio, por lo cual este parámetro es importante, además, del AVA para la toma de decisiones.

De todos los parámetros de severidad, el índice doppler demostró la correlación más fuerte con el AVA y el AVA indexada por ASC y la talla, independiente del tamaño de los anillos, por lo cual podría recomendarse su realización rutinaria en todos los pacientes como parámetro independiente de flujo. Estos hallazgos son similares a lo reportado 

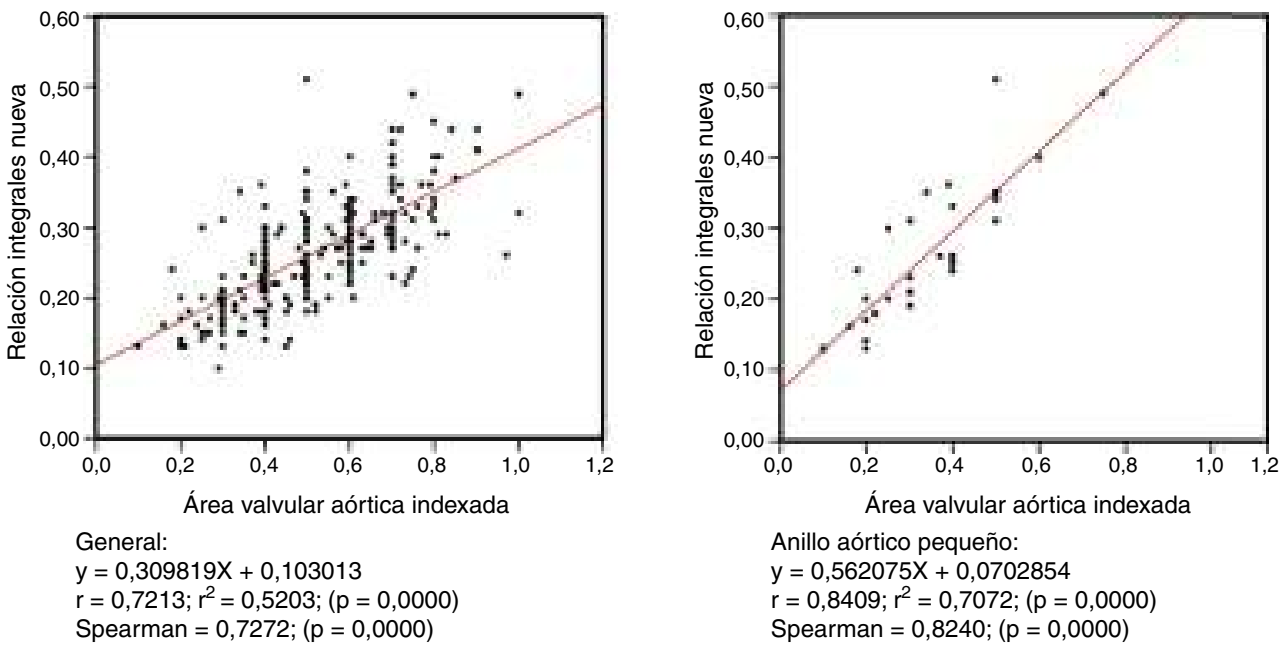

Anillo aórtico pequeño:

$y=0,562075 X+0,0702854$

$r=0,8409 ; r^{2}=0,7072 ;(p=0,0000)$

Spearman $=0,8240 ;(p=0,0000)$
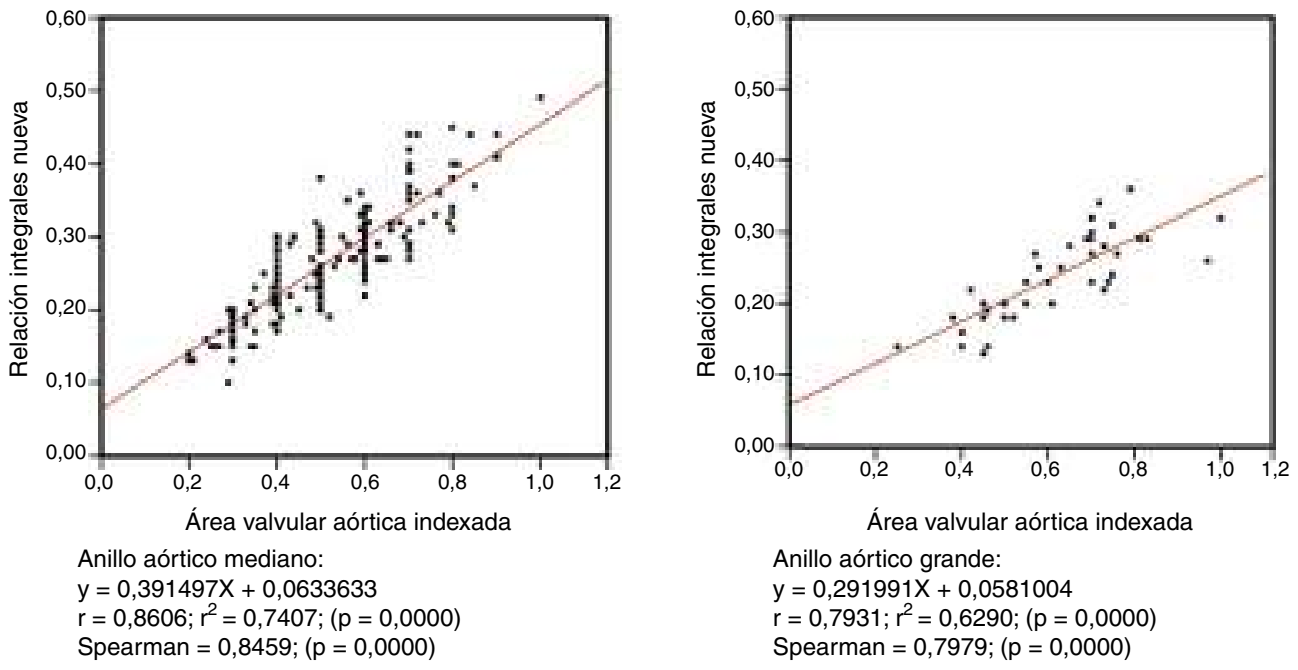

Figura 4 Correlación entre la relación de integrales (índice de velocidades doppler) y el área valvular aórtica indexada. El índice de velocidades doppler demostró fuerte correlación con el AVA indexada por ASC, independiente del tamaño del anillo.

por Michelena ${ }^{6}$, en donde se propone ajustar el punto de corte del índice doppler según el tamaño del anillo (tracto de salida del ventrículo izquierdo) $\mathrm{a} \leq, 0,35, \leq, 0,3 \mathrm{y} \leq$, 0,25 para pequeño, medianos y grandes, respectivamente. En nuestro estudio se encontró la mayor correlación para un área valvular aórtica de $<1,0 \mathrm{~cm}^{2}$ con un índice doppler de 0,4 para el anillo pequeño, 0,3 para el anillo mediano y 0,22 para los anillos grandes. Este hallazgo resalta aún más la necesidad de estudios encaminados a individualizar grupos específicos de pacientes al momento de clasificar el nivel de severidad, hasta ahora haciendo énfasis en parámetros como: sexo y diámetro del anillo aórtico o tracto de salida ventricular izquierdo.

El gradiente medio fue el parámetro que mostró el menor nivel de consistencia con el AVA aún indexada por ASC y la talla. Esto puede ser explicado por su alta dependencia del flujo y en parte por el grupo de pacientes con formas paradójicas de la estenosis aórtica (11,8\% de nuestra población), con gradientes más bajos. Los puntos de corte definidos por las guías aplican para pacientes con: fracción de eyección preservada y volumen sistólico normal.

Un porcentaje similar de formas paradójicas ha sido reportado en la literatura por otros autores que han demostrado que estos pacientes contribuyen de forma importante al grado de inconsistencias ${ }^{12}$, y por tanto, debe ser considerado como uno de los diagnósticos probables al afrontar esta circunstancia.

Este estudio no demostró diferencias significativas al correlacionar el AVA indexada por la superficie corporal en comparación con el AVA indexada por la talla con otros parámetros en los diferentes grupos de tamaño del anillo aórtico. Esto podría ser explicado en parte por el bajo número de pacientes con obesidad incluidos en nuestra población $(5,8 \%)$. En pacientes obesos ha sido demostrado que indexar el área valvular aórtica por ASC sobreestima la gravedad $^{13}$.

A partir de nuestros hallazgos es posible plantear la hipótesis de que la indexación por la talla puede ser válida para pacientes con obesidad, pues elimina el sesgo del peso en el cálculo del área de la superficie corporal. Esta hipótesis debe ser evaluada en estudios con mayor población para determinar los puntos de corte para el AVA indexada por la talla.

En resumen, los criterios definidos por las guías para determinar la severidad de la estenosis aórtica en pacientes con función sistólica normal, son inconsistentes, de esta 

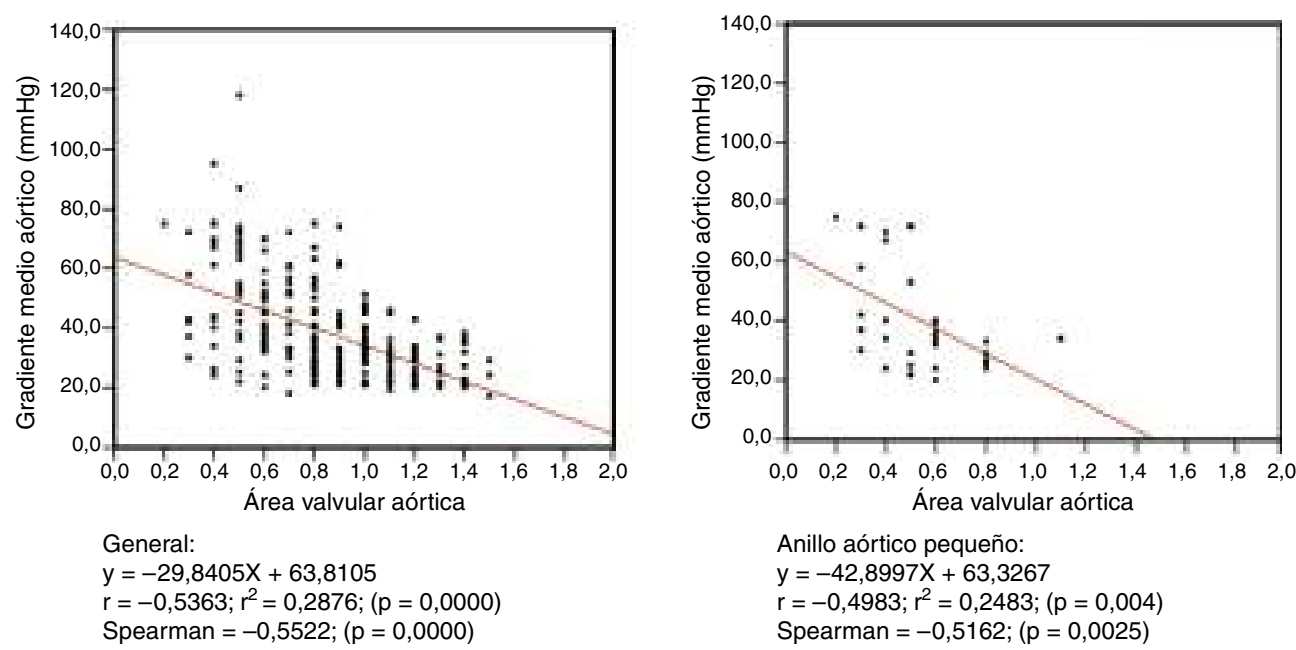

Anillo aórtico pequeño:

$y=-42,8997 X+63,3267$

$r=-0,4983 ; r^{2}=0,2483 ;(p=0,004)$

Spearman $=-0,5162 ;(p=0,0025)$
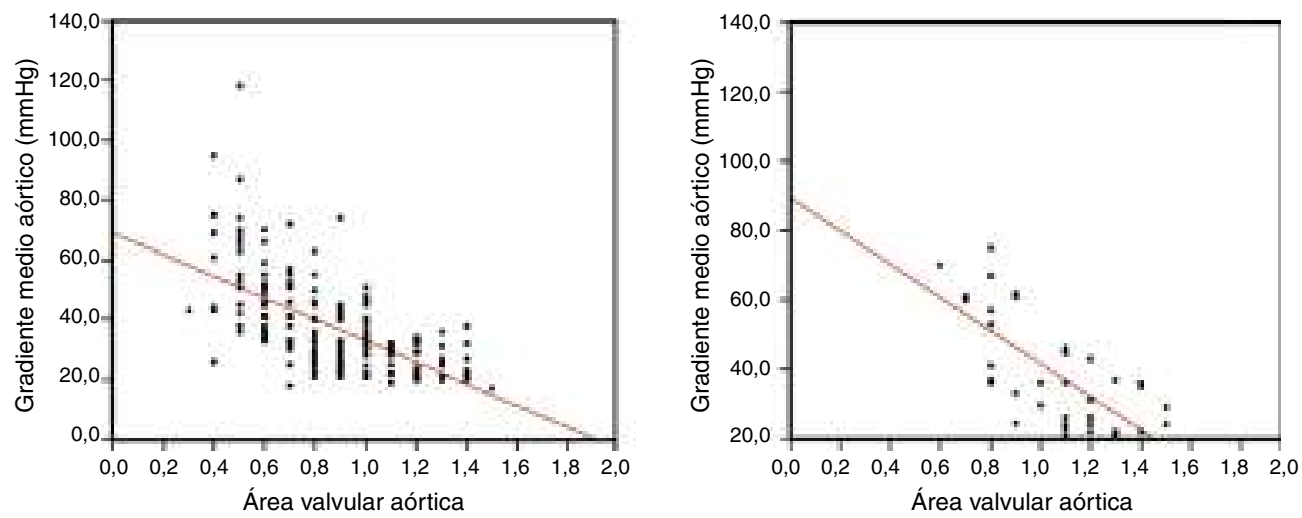

Anillo aórtico mediano: $y=-35,9573 X+68,8696$

$r=-0,5987 ; r^{2}=0,3584 ;(p=0,0000)$

Spearman $=-0,6351 ;(p=0,0000)$

Anillo aórtico grande:

$y=-47,7938 X+89,5204$

$r=-0,7080 ; r^{2}=0,5013 ;(p=0,0000)$

Spearman $=-0,6870 ;(p=0,0000)$

Figura 5 Correlación entre el gradiente medio aórtico y el área valvular aórtica. El AVA en relación con el gradiente medio demuestra variación significativa entre los diferentes grupos según el tamaño del anillo, siendo mayor la fuerza de correlación en el grupo de pacientes con anillo aórtico grande.

forma una proporción de pacientes que son clasificados en estenosis aórtica grave por el AVA demuestran una pobre correlación con otros parámetros, llevando a serias confusiones en el diagnóstico.

Varios autores ${ }^{6,14}$, han sugerido que un punto de corte del AVA $<0,8 \mathrm{~cm}^{2}$ puede ser mas apropiado, principalmente, en pacientes con el anillo pequeño y con bajo volumen sistólico, así este último punto de corte disminuiría el nivel de inconsistencia, especialmente, en pacientes con formas paradójicas de la estenosis aórtica.

Con la ecuación de continuidad para el cálculo del área valvular aórtica se asume el orifico aórtico como circular, lo cual se ha demostrado con la ecocardiografía tridimensional no es correcto, pues su forma es elíptica con una correlación débil entre los dos métodos $(K=0,36)$, según los hallazgos de Pérez de Isla y Cols. ${ }^{15}$. Esta es otra limitante importante en la evaluación de la gravedad de la estenosis aórtica con la ecuación de continuidad.

Las recomendaciones de las guías actuales muestran mayor consistencia para pacientes con anillos medianos y grandes, por lo que la utilización de otros parámetros como el índice doppler pueden aportar más elementos para la clasificación de la gravedad y la toma de decisiones clínicas. En este estudio la indexación del AVA por el ASC y la talla no demostró: diferencias significativas, ni disminuyó las inconsistencias en pacientes con el anillo aórtico pequeño, como lo han sugerido las guías ${ }^{2,3}$.

\section{Limitaciones del estudio}

Se trata de un estudio observacional, descriptivo en el cual se evaluaron las variables demográficas y ecocardiográficas, no se evaluaron desenlaces clínicos ni seguimiento de los pacientes para definir el pronóstico en mortalidad y necesidad de cambio valvular aórtico.

No se exploró como se alteran las correlaciones con un punto de corte del AVA $<0,8 \mathrm{~cm}^{2}$ para definir la estenosis aórtica grave, como lo han sugerido otros autores.

No se analizó en forma independiente el grupo de la estenosis aórtica grave con bajo flujo, bajo gradiente por el pequeño número de pacientes $(\mathrm{N}=29,11,8 \%)$. 


\section{Conclusiones}

Los criterios y parámetros empleados para clasificar la gravedad de la estenosis aórtica definidos por las guías internacionales son inconsistentes. Si se tiene en cuenta solo el área valvular aórtica se sobreestima la gravedad en comparación con otros parámetros, principalmente, en el grupo de pacientes con el anillo aórtico pequeño. El grado de inconsistencia es menor en pacientes con el anillo mediano y grande.

Además, del anillo aórtico otra fuente de inconsistencias son: las formas paradójicas de la estenosis aórtica y representan entre el $10-15 \%$ de la población de pacientes con la estenosis aórtica grave, por lo cual es importante su reconocimiento.

Se requieren más estudios para evaluar puntos de corte más consistentes con los parámetros actuales y explorar si el punto de corte de la estenosis aórtica grave por el área valvular aórtica menor o igual de $0.8 \mathrm{~cm}^{2}$ es el más apropiado.

\section{Responsabilidades éticas}

Protección de personas y animales. Los autores declaran que para esta investigación no se han realizado experimentos en seres humanos ni en animales.

Confidencialidad de los datos. Los autores declaran que en este artículo no aparecen datos de pacientes.

Derecho a la privacidad y consentimiento informado. Los autores declaran que en este artículo no aparecen datos de pacientes.

\section{Conflicto de intereses}

Los autores declaran no tener ningún conflicto de intereses.

\section{Agradecimientos}

A los doctores: Daniel Piñeiro, Marcia Barbosa, Ricardo Migliore y Héctor Michelena por la revisión del trabajo y las sugerencias previas a esta publicación.

\section{Bibliografía}

1. Everborn GW, Schirmer H, Heggelund G, Lunde P, Ramussen K. The evolving epidemiology of valvular aortic stenosis. Heart. 2013;99:396-400.
2. Nishimura RA, Otto CM, Bonow RO, Carabello BA, Erwin JP, Guyton RA, et al. 2014 AHA/ACC guideline for the management of patients with valvular heart disease: a report of the American College of Cardiology/American Heart Association Task Force on Practice Guidelines. J Am Coll Cardiol. 2014 Jun 10;63:e57-185.

3. Vahanian A, Alfieri O, Andreotti F, Antunes MJ, Barón-Esquivias $\mathrm{G}$, Baumgartner $\mathrm{H}$, et al. Guidelines on the management of valvular heart disease (version 2012). Eur Heart J. 2012 Oct;33:2451-96.

4. Minners J, Allgeier M, Gohlke-Baerwolf C, Kienzle RP, Neumann FJ, Jander N. Inconsistencies of echocardiographic criteria for the grading of aortic valve stenosis. Eur Heart J. 2008 Apr;29:1043-8.

5. Minners J, Allgeier M, Gohlke-Baerwolf C, Kienzle RP, Neumann FJ, Jander N. Inconsistent grading of aortic valve stenosis by current guidelines: haemodynamic studies in patients with apparently normal left ventricular function. Heart. 2010 Sep;96:1463-8.

6. Michelena HI, Margaryan E, Miller FA, Eleid M, Maalof J, Rakesh $\mathrm{S}$, et al. Inconsistent echocardiographic grading of aortic stenosis: is the left ventricular outflow tract important? Heart. 2013 Jul;99:921-31.

7. Galan A, Zoghbi WA, Quiñonez MA. Determination of severity of valvular aortic stenosis by doppler echocardiography and relation of findings to clinical outcome and agreement with hemodinamyc measurements determined at cardiac catetherization. Am J Cardiol. 1991;67:1007-12.

8. Baumgertner H, Hung J, Bermejo J, Chambers JB, Evangelista A, Griffin BP, et al. Echocardiographic assessment of valve stenosis: EAE/ASE recomendations for clinical practice. J Am Soc Echocardiogr. 2009;22:1-23.

9. Pibarot P, Garcia D, Dumesnil JG. Energy loss index in aortic stenosis: from fluid mechanics concept to clinical application. Circulation. 2013;127:1101-4.

10. Lancellotti P, Magne J. Valvuloarterial Impedance in aortic stenosis: look at the load, but do not forget the flow. Eur J Echocardiogr. 2011;5:354-7.

11. Lancellotti P, Lebois F, Simon M, Tombeux C, Chauvel C, Pierard LA. Prognostic importance of quantitative exercise Doppler echocardiography in asymptomatic valvular aortic stenosis. Circulation. 2005;112 Suppl. 9:1377-82.

12. Hachicha Z, Dumesnil JG, Bogaty P, Pibarot P. Paradoxical low-flow, low-gradient severe aortic stenosis despite preserved ejection fraction is associated with higher afterload and reduced survival. Circulation. 2007;115:2856-64.

13. Rogge BP, Gerdts E, Cramariuc D, Bahlmann E, Jander N, Gohlke-Bärwolf C. Impact of obesity and nonobesity on grading the severity of aortic valve stenosis. Am J Cardiol. 2014 May 1;113:1532-5.

14. Pibarot P, Dumesnil JG. Improving assessment of aortic stenosis. J Am Coll Cardiol. 2012 Jul 17;60:169-80.

15. Pérez de Isla L, Zamorano J, Pérez de Iglesia R, Ciocarelli $\mathrm{S}$, Almería C, Rodrigo J, et al. Quantification of aortic valve area using three-dimensional echocardiography. Rev Esp Cardiol. 2008 May;61:494-500. 